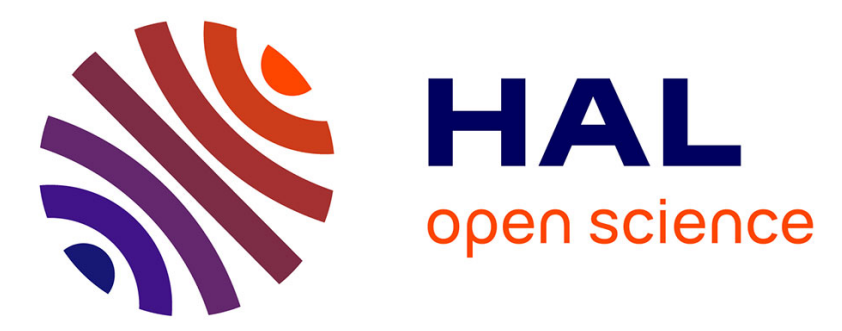

\title{
State-to-state inelastic rotational cross sections in five-atom systems with the multiconfiguration time dependent Hartree method
}

\author{
Steve Ndengue, Yohann Scribano, Fabien Gatti, Richard Dawes
}

\section{To cite this version:}

Steve Ndengue, Yohann Scribano, Fabien Gatti, Richard Dawes. State-to-state inelastic rotational cross sections in five-atom systems with the multiconfiguration time dependent Hartree method. Journal of Chemical Physics, 2019, 151 (13), pp.134301. 10.1063/1.5119381 . hal-02179642

\section{HAL Id: hal-02179642 \\ https://hal.science/hal-02179642}

Submitted on 14 Dec 2020

HAL is a multi-disciplinary open access archive for the deposit and dissemination of scientific research documents, whether they are published or not. The documents may come from teaching and research institutions in France or abroad, or from public or private research centers.
L'archive ouverte pluridisciplinaire $\mathbf{H A L}$, est destinée au dépôt et à la diffusion de documents scientifiques de niveau recherche, publiés ou non, émanant des établissements d'enseignement et de recherche français ou étrangers, des laboratoires publics ou privés. 


\title{
State-to-state inelastic rotational cross sections in five-atom systems with the MCTDH method
}

\author{
Steve Ndengué, ${ }^{1,2}$ Yohann Scribano, ${ }^{3}$ Fabien Gatti, ${ }^{4}$ and Richard Dawes ${ }^{2}$ a) \\ ${ }^{1)}$ ICTP-East African Institute for Fundamental Research, University of Rwanda, Kigali, \\ Rwanda \\ ${ }^{2)}$ Department of Chemistry, Missouri University of Science and Technology, 65409 Rolla, Missouri, \\ United States. \\ 3) Laboratoire Univers et Particules de Montpellier, Université de Montpellier, \\ LUPM - UMR CNRS 5299, Place Eugène Bataillon, 34095 Montpellier Cedex, \\ France. \\ ${ }^{4)}$ Institut de Sciences Moleculaires d'Orsay, UMR 8214, Université Paris-Sud - Université Paris-Saclay, \\ 91405 Orsay, France.
}

(Dated: 9 July 2019)

We present a MCTDH method as an attractive alternative approach to the usual quantum close-coupling method which approaches some computational limits in the calculation of rotational excitation (and deexcitation) between polyatomic molecules (here collisions between triatomic and diatomic rigid molecules). We have perfomed a computational investigation of the rotational (de-)excitation of the benchmark rigid rotor $\mathrm{H}_{2} \mathrm{O}-\mathrm{H}_{2}$ system on a recently developed Potential Energy Surface of the complex using the MultiConfiguration Time Dependent Hartree (MCTDH) method. We focus here on excitations and de-excitations from the $0_{00}$, $1_{11}$ and $1_{10}$ states of $\mathrm{H}_{2} \mathrm{O}$ with $\mathrm{H}_{2}$ in its ground rotational state, looking at all the potential transitions in the energy range $1-200 \mathrm{~cm}^{-1}$. This work follows a recently completed study on the $\mathrm{H}_{2} \mathrm{O}-\mathrm{H}_{2}$ cluster where we characterized its spectroscopy, and more generally serves a broader goal to describe inelastic collision processes of high dimensional systems using the MCTDH method. We find that the cross sections obtained from the MCTDH calculations are in excellent agreement with time independent calculations from previous studies, but does become challenging for the lower kinetic energy range of the de-excitation process. The MCTDH method therefore appears to be a useful complement to standard approaches to study inelastic collision for various collision partners, even at low energy, though performing better for rotational excitation than for de-excitation.

\section{INTRODUCTION}

Our understanding of energy transfer between colliding atoms and molecules is crucial for several fields like chemical processes in Earth's atmosphere, combustion chemistry processes for industrial and aeronautic applications, and also in outside Earth's environnement, such as in the InterStellar Medium (ISM), stellar and planetary (and exo-planetary) atmospheres. For example, determination of molecular abundances in proto-stellar clouds requires one to solve radiative transfer equations which themselves require knowledge of state-to-state rotational excitation (and de-excitation) rate constants. The most accurate technique to determine accurate rotational inelastic cross sections (and thus rate constants) is certainly the time independent quantum method based on Close Coupling (CC) which solves the nuclear Schrödinger equation. The CC-methodology is a powerful and intuitive method to solve for the rovibrational states and obtain the collisional cross-sections using potential energy function typically obtained by means of accurate $a b$ initio methods. This approach was intensively used in the past for the simulation of rotationally inelastic collisions of nonreactive diatomic and polyatomic molecules with

\footnotetext{
a)dawesr@mst.edu
}

atoms and molecular colliders such as $\mathrm{H}_{2}$ which is the most abundant molecule in space. The other well-known technique is the Quasi-Classical trajectory method which solves the classical Hamilton's equations of motion, and is usually processed by quantizing the classical action of trajectories using binning histogram methods. This approach is numerically very efficient since computational time decreases as the collision energy increases. However, the QCT approach is best suited for the high temperature regime where quantum effects are expected to be less important, and whereas for low temperature, its validity could be questionable. More recently, the mixed quantum-classical trajectory $(\mathrm{MQCT})^{1}$ and statistical adiabatic channel model $(\mathrm{SACM})^{2,3}$ have been proposed as alternative approaches to the $\mathrm{CC}$ method and they have both shown very promising results in certain cases, but less accurate behavior in other cases, with their domain of validity not yet fully known and needing to be investigated.

In this work, we want to address another alternative approach which can have some numerical advantages compared to CC approaches and especially when we consider high-dimensional molecular systems for which the density of internal rovibrationnal state is large leading to unfeasable CC simulations. Here we extend a recently reported MCTDH investigation ${ }^{4}$ of $\mathrm{Ar}$ colliding with a rigid triatomic rotor $\left(\mathrm{H}_{2} \mathrm{O}\right)$ by studying the collision between diatomic rigid rotors with triatomic rigid rotors using 
the MCTDH methodology. This five-dimensional system corresponds to the description of rigid rotors inelastic rotational excitation, an example of which is $\mathrm{H}_{2} \mathrm{O}$ colliding with $\mathrm{H}_{2}$, a system of interest for ISM and astrochemistry. In the past, the spectroscopy of the weakly bounded complex $\mathrm{H}_{2} \mathrm{O}-\mathrm{H}_{2}$ was intensively studied ${ }^{5-9}$ but also the collisional dynamics between those two molecules with various methods. Those methods used Time Independent CC formalism ${ }^{7,10-15}$, as well as quasi-classical approach ${ }^{16}$ which make this system a very good five-dimensional benchmark. The MCTDH method has emerged in recent years as one of the methods of choice for the study of the quantum molecular dynamics (spectroscopy or collisional dynamics) for system in high dimensionality. We are particularly focused here on the low-energy collision region in order to most stringently test the method. This is because of the resonance structures mentioned before, and also because of the known difficulty for time-dependent approaches to describe low-energy collisions, since the wavepacket in that case moves slowly, and the propagation then takes a very long time (which may lead to a propagation of errors).

The paper is organized as follows: in Sec.II we will first give a brief introduction to the MCTDH method and the Hamiltonian (the kinetic energy operator and the potential energy surfaces) used for the dynamics, in Sec.IID we will describe how the cross-sections are obtained and discuss the convergence of the calculations. In Sec.III we will present our results and their comparison to CC simulations. We will finally conclude in Sec.IV and give some perspectives of this method for more complex molecular systems.

\section{COMPUTATIONAL METHODOLOGY}

\section{A. MCTDH method}

The MultiConfiguration Time Dependent Hartree $(\mathrm{MCTDH})^{17-20}$ is a time-dependent method in which each degree of freedom is associated with a small number of orbitals or single particle functions (SPFs) which, through their time dependence, allow an efficient description of the molecular dynamic process. The total MCTDH wave function is expanded in Hartree products, that is, products of single-particle functions

$$
\begin{aligned}
\Psi\left(Q_{1}, \ldots, Q_{f}, t\right) & =\sum_{j_{1}=1}^{n_{1}} \cdots \sum_{j_{f}=1}^{n_{f}} A_{j_{1} \cdots j_{f}}(t) \prod_{\kappa=1}^{f} \varphi_{j_{\kappa}}^{(\kappa)}\left(Q_{\kappa}, t\right) \\
& =\sum_{J} A_{J} \Phi_{J}
\end{aligned}
$$

where $f$ is the number of degree of freedom of the system, $Q_{1}, \ldots, Q_{f}$ are the nuclear coordinates, $A_{j_{1} \cdots j_{f}}$ denotes the MCTDH expansion coefficients, and $\varphi_{j_{\kappa}}^{(\kappa)}\left(Q_{\kappa}, t\right)$ are the $n_{\kappa}$ SPFs associated with each degree of freedom $\kappa$. The subsequent equation of motion for the coefficients and single particle functions, obtained by applying the Dirac-Frenkel variational principle to the wave function ansatz, are a set of coupled nonlinear differential equations which conserve the norm and the total energy (for time-independent Hamiltonian).

The MCTDH equations of motion are solved at every time step and require to build the mean fields and the Hamiltonian matrix. As the Hamiltonian matrix and mean fields require evaluation of integrals of the type

$$
\left\langle\varphi_{j_{1}}^{(1)} \cdots \varphi_{j_{f}}^{(f)}|\hat{H}| \varphi_{l_{1}}^{(1)} \cdots \varphi_{l_{f}}^{(f)}\right\rangle
$$

Because of the considerable time those calculations take in the process, the MCTDH approach relies in transforming the Hamiltonian operator $\hat{H}$ to a sum-of-products of single particle operators

$$
\hat{H}=\sum_{r=1}^{s} c_{r} \prod_{\kappa=1}^{f} \hat{h}_{r}^{(\kappa)}
$$

which then significantly simplifies the evaluation of the Hamiltonian matrix and the mean fields

$$
\left\langle\varphi_{j_{1}}^{(1)} \cdots \varphi_{j_{f}}^{(f)}|\hat{H}| \varphi_{l_{1}}^{(1)} \cdots \varphi_{l_{f}}^{(f)}\right\rangle=\sum_{r=1}^{s} c_{r} \prod_{\kappa=1}^{f}\left\langle\varphi_{j_{\kappa}}^{(\kappa)}\left|\hat{h}_{r}^{(\kappa)}\right| \varphi_{l_{\kappa}}^{(\kappa)}\right\rangle .
$$

In the following two subsections we will describe the expression of the Kinetic Energy Operator and the Potential Energy Operator as a sum-of-products of single particle operators.

\section{B. MCTDH Kinetic Energy Operator}

We described quite extensively in the previous paper ${ }^{21}$ on this system the Hamiltonian (Kinetic Energy Operator (KEO) and Potential Energy Surface (PES)). The Kinetic Energy Operator (KEO) expressed in Jacobi coordinates is used in this work, following the subsystem KEO derivation presented by Brocks et $a .^{22}$ and generalized by Gatti and $\mathrm{Iung}^{23}$. Here, just as in our previous work $^{21}$ on the same system we work in the $\mathrm{E}_{2}$ frame obtained by rotation of the first two Euler angles of the SF frame. Althrough this section we express the KEO in atomic units.

$$
\begin{aligned}
2 \hat{T} & =-\frac{1}{\mu_{R}} \frac{\partial^{2}}{\partial R^{2}}+2 \hat{T}_{1}+2 \hat{T}_{2} \\
& +\frac{1}{\mu R^{2}}\left(\hat{\vec{J}}^{\dagger} \hat{\vec{J}}+\left(\hat{\vec{L}}_{1}+\hat{\vec{L}}_{2}\right)^{2}-2\left(\hat{\vec{L}}_{1}+\hat{\vec{L}}_{2}\right) \hat{\vec{J}}\right)_{E_{2}}
\end{aligned}
$$

where $\mu_{R}$ is the reduced mass of the $\mathrm{H}_{2} \mathrm{O}-\mathrm{H}_{2}$ cluster, the 1 and 2 subscripts refer respectively to the $\mathrm{H}_{2} \mathrm{O}$ and $\mathrm{H}_{2}$ fragments. The rigid rotor Hamiltonian of the $\mathrm{H}_{2} \mathrm{O}$ 
molecule is expressed as $^{22,24}$

$$
\begin{aligned}
\hat{T}_{1} & =\frac{A}{2}\left(\hat{L}_{1,+}^{2}+\hat{L}_{1,-}^{2}+\hat{L}_{1,+} \hat{L}_{1,-}+\hat{L}_{1,-} \hat{L}_{1,+}\right)_{B F_{1}} \\
& -\frac{C}{2}\left(\hat{L}_{1,+}^{2}+\hat{L}_{1,-}^{2}-\hat{L}_{1,+} \hat{L}_{1,-}-\hat{L}_{1,-} \hat{L}_{1,+}\right)_{B F_{1}} \\
& +B \hat{L}_{z^{B F_{1}}}^{2},
\end{aligned}
$$

where the rotational constants ${ }^{6}$ have values $A=27.8572$ $\mathrm{cm}^{-1}, B=14.5145 \mathrm{~cm}^{-1}$ and $C=9.2799 \mathrm{~cm}^{-1}$. The rigid rotor kinetic energy of the $\mathrm{H}_{2}$ fragment is written simply as $\hat{T}_{2}=B_{H_{2}} \hat{\vec{L}}_{2}^{2}$ where the rotational constant $B_{H_{2}}=59.2434 \mathrm{~cm}^{-1}$. The final form of the KEO obtained after some analytical development and which is implemented in the MCTDH code is then

$$
\begin{aligned}
2 \hat{T} & =-\frac{1}{\mu_{R}} \frac{\partial^{2}}{\partial R^{2}}+2 \hat{T}_{1}+2 \hat{T}_{2} \\
& +\frac{1}{\mu R^{2}}\left(J(J+1)+\hat{\vec{L}}_{1}^{2}+\hat{\vec{L}}_{2}^{2}-2 \hat{L}_{1, z}^{2}-2 \hat{L}_{2, z}^{2}\right)_{E_{2}} \\
& +\frac{1}{\mu R^{2}}\left(\hat{L}_{1,+} \hat{L}_{2,-}+\hat{L}_{1,-} \hat{L}_{2,+}-2 \hat{L}_{1, z} \hat{L}_{2, z}\right)_{E_{2}} \\
& +\frac{1}{\mu R^{2}}\left(C_{+}(J, K)\left(\hat{L}_{1,+}+\hat{L}_{2,+}\right)\right)_{E_{2}} \\
& +\frac{1}{\mu R^{2}}\left(C_{-}(J, K)\left(\hat{L}_{1,-}+\hat{L}_{2,-}\right)\right)_{E_{2}}
\end{aligned}
$$

with

$$
C_{ \pm}(J, K)=\sqrt{(J(J+1)-K(K \pm 1)} .
$$

In the previous equations, $\hat{\vec{L}}_{X}(X=1,2)$ is the total angular momentum of fragment 1 or 2 and $\hat{L}_{X, \pm}$ are their corresponding creating and lowering operators expressed as $\hat{L}_{X, \pm}=\hat{L}_{X, x} \pm \hat{L}_{X, y}$. In terms of angles, we can write for their expressions in the $E_{2}$ frame

$$
\begin{gathered}
\hat{L}_{1}^{2}=-\frac{1}{\sin \beta} \frac{\partial}{\partial \beta} \sin \beta \frac{\partial}{\partial \beta}+\frac{k_{\alpha}}{\sin ^{2} \beta}, \\
\hat{L}_{2}^{2}=-\frac{1}{\sin \theta} \frac{\partial}{\partial \theta} \sin \theta \frac{\partial}{\partial \theta}+\frac{k_{\phi}}{\sin ^{2} \theta}
\end{gathered}
$$

and

$$
\begin{aligned}
& \hat{L}_{1 \pm}= \pm \frac{\partial}{\partial \beta}-k_{\alpha} \cot \beta \\
& \hat{L}_{2 \pm}= \pm \frac{\partial}{\partial \theta}-k_{\phi} \cot \theta
\end{aligned}
$$

with an additional shift $k \rightarrow k \pm 1$ in the equations (11) and (12). In the $\mathrm{BF}_{1}$ frame (for $\mathrm{H}_{2} \mathrm{O}$ ) we have similar expressions with $k_{\alpha}$ changed into $k_{\gamma}$ and the corresponding additional shift $k_{\gamma} \rightarrow k_{\gamma} \pm 1$

$$
\hat{L}_{1}^{2}=-\frac{1}{\sin \beta} \frac{\partial}{\partial \beta} \sin \beta \frac{\partial}{\partial \beta}+\frac{k_{\gamma}}{\sin ^{2} \beta}
$$

and

$$
\hat{L}_{1 \pm}= \pm \frac{\partial}{\partial \beta}-k_{\gamma} \cot \beta
$$

\section{MCTDH Potential Energy Operator}

We use as in our previous work ${ }^{21}$ the same PES of Valiron et $a l^{25}$ and transform it to the appropriate set of coordinates $^{21}$, but now for scattering calculations. Using the multipolar description of Valiron PES and the transformation suggested by Avoird and Nesbitt ${ }^{5}$, we can have an analytically exact representation of the PES in the set of coordinates used for the dynamics and thus be able to extend the PES to any distance without doing an additional refitting with MCTDH. This analytical representaion is particularly useful as the range of the PES which is significantly extended along the $R$ coordinate in order to describe accurately the low energy region of the cross section. The surface from Ref. 25 after transformation from its original set of coordinates to the $E_{2}$ frame can be expressed as

$$
V\left(R, \beta_{1}, \gamma_{1}, \alpha_{1}, \theta_{2}, \phi_{2}\right)=\sum_{\substack{r_{\beta}, r_{\gamma} \\ r_{\alpha}, r_{\theta}}} \underset{r_{\alpha}, r_{\theta}}{\tilde{r}_{r_{\beta}, r_{\gamma}}}(R) \underset{r_{\alpha}, r_{\theta}}{r_{r_{\beta}, r_{\gamma}}}\left(\omega_{1}, \omega_{2}\right)
$$

where

$$
\underset{r_{\alpha}, r_{\theta}}{f_{r_{\beta}, r_{\gamma}}}\left(\omega_{1}, \omega_{2}\right)=D_{r_{\alpha}, r_{\gamma}}^{\left(r_{\beta}\right)}\left(\beta_{1}, \gamma_{1}, \alpha_{1}\right)^{\star} C_{r_{\theta},-r_{\alpha}}\left(\theta_{2}, \phi_{2}\right) .
$$

$D_{r_{\alpha}, r_{\gamma}}^{\left(r_{\beta}\right)}\left(\beta_{1}, \gamma_{1}, \alpha_{1}\right)$ is the Wigner D-matrix and $C_{r_{\theta},-r_{\alpha}}\left(\theta_{2}, \phi_{2}\right)$ is the Racah normalized spherical harmonics. As we work in the momentum representation for the degrees of freedom $\gamma, \alpha$ and $\phi$ we replace $\gamma_{1}, \alpha_{1}$ and $\phi_{1}$ by their momentum representation $k_{\gamma}, k_{\alpha}$ and $k_{\phi}$ where we dropped the 1 and 2 indices to simplify the notation.

\section{Inelastic cross-sections calculations}

The $\mathrm{H}_{2} \mathrm{O}+\mathrm{H}_{2}$ scattering calculations were designed in a similar way as our previous scattering studies ${ }^{4,9,26}$ where we used the MCTDH program: we will repeat those steps here.

(i) First a wavepacket defining the initial state has to be constructed: it is here (since we are in the rigid rotor approximation) the product of the initial rotational state of $\mathrm{H}_{2} \mathrm{O}$, the initial rotational state of $\mathrm{H}_{2}$ and a Gaussian function along the dissociative coordinate $R$, starting far away from the interaction region. The Gaussian's parameters (with a negative impulsion) are selected in order to cover the energy range of interest. The initial wavefunction is thus expressed as

$$
\begin{aligned}
\Psi_{i}\left(R, \beta, k_{\gamma}, k_{\alpha}, \tilde{\theta}, k_{\phi}\right) & =\psi_{i}\left(\beta, k_{\gamma}, k_{\alpha}, \tilde{\theta}, k_{\phi}\right) \chi(R) \\
& =\tau_{1, i}\left(\beta, k_{\gamma}, k_{\alpha}\right) \tau_{2, i}\left(\theta, k_{\phi}\right) \chi(R)
\end{aligned}
$$

where $\psi_{i}$ is a product of the initial rotation states of $\mathrm{H}_{2} \mathrm{O}\left(\tau_{1, i}\right)$ and $\mathrm{H}_{2}\left(\tau_{2, i}\right)$. The initial rotational state of $\mathrm{H}_{2} \mathrm{O}$ are linear combination of Wigner-D matrices usually written in the simplified notation $j_{K_{a} K_{c}}$ where $j$ is the molecular rotational angular momentum and $K_{a}, K_{c}$ the 
TABLE I. Rotational energy levels $\left(\mathrm{cm}^{-1}\right)$ or ortho and para $\mathrm{H}_{2} \mathrm{O}$ and $\mathrm{H}_{2}$. The rotational constants used to generate those levels are $A=27.8572 \mathrm{~cm}^{-1}, B=14.5145 \mathrm{~cm}^{-1}$ and $C=9.2799$ $\mathrm{cm}^{-1}$ for $\mathrm{H}_{2} \mathrm{O}$ and $B_{\mathrm{H}_{2}}=59.2434 \mathrm{~cm}^{-1}$ for $\mathrm{H}_{2}$. Ortho levels of $\mathrm{H}_{2}$ are displayed in italic.

\begin{tabular}{cccccc}
\hline \multicolumn{2}{c}{ ortho } & \multicolumn{2}{c}{ para } & \multicolumn{2}{c}{$\begin{array}{c}\mathrm{H}_{2} \\
\text { ortho \& para }\end{array}$} \\
\hline $1_{01}$ & 23.7995 & $0_{00}$ & 0.00000 & 0 & 0.0000 \\
$1_{10}$ & 42.4024 & $1_{11}$ & 37.1583 & 1 & 118.4868 \\
$2_{12}$ & 79.5133 & $2_{02}$ & 70.1329 & 2 & 355.4604 \\
$2_{21}$ & 135.3220 & $2_{11}$ & 95.2454 & 3 & 710.9208 \\
$3_{12}$ & 173.5976 & $2_{20}$ & 136.5876 & 4 & 1184.8680 \\
\hline
\end{tabular}

projection on the BF-axis along the prolate and oblate limits. If, for example, we write the Wigner D-matrices as $|j, k, m\rangle$, the rotational state for $j=0$ is $\left|0_{00}\right\rangle=|0,0,0\rangle$ with eigenvalue 0 . The rotational states for $j=1$ are $^{6}$ :

$$
\begin{aligned}
& \left|1_{10}\right\rangle=\frac{1}{\sqrt{2}}[|1,1, m\rangle+|1,-1, m\rangle], \\
& \left|1_{11}\right\rangle=|1,0, m\rangle, \\
& \left|1_{01}\right\rangle=\frac{1}{\sqrt{2}}[|1,1, m\rangle-|1,-1, m\rangle],
\end{aligned}
$$

with respective eigenvalues $A+B, A+C$ and $B+C$ (clearly with a $2 j+1=3$ degeneracy) with $A, B$ and $C$ being the rotational constants of $\mathrm{H}_{2} \mathrm{O}$ given above and $m=-1,0,1$ the magnetic quantum number projections. For $j \geq 2$, we used the symbolic arithmetic program Sympy $^{27}$ and obtained the normalized eigenfunctions and related eigenvalues that helped to generate analytically the initial but also the final rotational functions. While it is obviously not compulsory to obtain the initial and final functions analytically, it nevertheless helps to verify that these results match the known analytical results for this rotational Hamiltonian.

The initial state of $\mathrm{H}_{2}$ is an associated Legendre polynomial that we write simply as $l_{m}$ where $l$ is the molecular angular momentum of the $\mathrm{H}_{2}$ fragment and $m$ the projection along the BF-axis. The Gaussian $\chi$ writes

$$
\chi(R)=\frac{1}{\sqrt{2 \pi w}} \exp \left[-\left(\frac{R-R_{0}}{2 w}\right)^{2}\right] e^{i p_{0}\left(r-R_{0}\right)}
$$

with $R_{0}, p_{0}$ and $w$ being respectively its center in coordinate and momentum space and its width. For this work, we tested various parameters and two sets of energy distributions (for which a state-to-state cross section will be presented later) are shown in Figure 2.

When the initial rotational states of $\mathrm{H}_{2} \mathrm{O}$ or $\mathrm{H}_{2}$ have a non-zero angular quantum number $\left(j \neq 0\right.$ in $j_{K_{a} K_{c}}$ or $l \neq 0$ in $\left.l_{m}\right)$, then calculations for each magnetic quantum number projection have to be done also in order to obtain the state-to-state cross-sections from a specific initial rotational state. The lowest para and ortho rotational levels of $\mathrm{H}_{2} \mathrm{O}$ and $\mathrm{H}_{2}$ are presented in Table I. The $2 j+1$ (and $2 l+1$ ) repetition of the calculations is avoided
TABLE II. Parameters of the primitive basis used for the rovibrational calculations of $\mathrm{H}_{2} \mathrm{O}-\mathrm{H}_{2}$. FFT stands for the

\begin{tabular}{|c|c|c|c|c|}
\hline Coordinate & $\begin{array}{c}\text { Primitive } \\
\text { Basis }\end{array}$ & $\begin{array}{c}\text { Number of } \\
\text { Points }\end{array}$ & Range & $\begin{array}{c}\text { Size of } \\
\text { SPF basis }\end{array}$ \\
\hline $\mathrm{R}$ & FFT & 960 & $2.0-72.0$ & $10-20$ \\
\hline$\beta$ & Wigner & 6 & $0-5$ & $10-25$ \\
\hline $\mathrm{k}_{\gamma}$ & K & 11 & $-5-5$ & \\
\hline $\mathrm{k}_{\alpha}$ & $\mathrm{K}$ & 11 & $-5-5$ & \\
\hline$\tilde{\theta}$ & KLeg & 3 & $0-2$ & $10-12$ \\
\hline $\mathrm{k}_{\phi}$ & $\mathrm{K}$ & 5 & $-2-2$ & \\
\hline
\end{tabular}
Fast Fourier Transform. Wigner stands for the Wigner DVR. KLeg is the K-Legendre DVR. The units for distance and angle are bohrs and radians respectively.

due to the symmetry of $\mathrm{H}_{2} \mathrm{O}$ (and $\mathrm{H}_{2}$ ). If we write an initial rotational eigenstate of $\mathrm{H}_{2} \mathrm{O}$ as $|j, \zeta, m\rangle\left(\mathrm{H}_{2}\right.$ as $|l, m\rangle)$, then the state-to-state probabilities from the initial states $|j, \zeta, m\rangle$ and $|j, \zeta,-m\rangle(|l, m\rangle$ and $|l,-m\rangle)$ are identical and instead of running for a rotational state $j_{K_{a} K_{c}}$ of $\mathrm{H}_{2} \mathrm{O}$ and $l$ of $\mathrm{H}_{2}(2 j+1) \times(2 l+1)$ calculations, we can simply run $(j+1) \times(l+1)$ calculations to obtain the cross-sections.

(ii) Next we have to use a suitable Complex Absorbing Potential (CAP). The intensity of the CAP was determined using the program plcap included in the MCTDH package. As before the form of the CAP is $W(x)=\eta\left|x-x_{c}\right|^{b} \Theta\left(x-x_{c}\right)$, where $\Theta$ is the Heaviside step function, $x_{c}$ the starting point of the CAP was selected such that the length of the CAP is 20 bohrs, $\eta$ its strength is $4.76 \times 10^{-9}$ and $b$ its order is 4 .

(iii) We then have to select a primitive and SPF bases for the calculation. The primitive basis, its range and the number of the SPFs used for the wavepacket propagation are summarized in Table II. A primitive basis composed of Fast Fourier Transform (FFT) functions for the intermolecular distance $R$ was coupled with a Wigner-DVR ${ }^{24}$ and a two dimensional Legendre-K $\mathrm{DVR}^{28}$ to describe respectively the orientation of the $\mathrm{H}_{2} \mathrm{O}$ and the $\mathrm{H}_{2}$ fragments in the $E_{2}$ frame.

(iv) As we are interested in the low-energy region of the cross-sections, we need to propagate the wavepacket for a very long time. In order to control the propagation and the convergence of the calculation, we allowed the wave packet to propagate for a maximum of $200 \mathrm{ps}$ (with the wavefunction printed every $20 \mathrm{fs}$ ) or when $99.0 \%$ of the wavepacket has been absorbed by the CAP. For higher values of $J$ the propagations completes very quickly: for example, for $J=35$ the calculation stops after about 10 ps while for $J=0$ a complete 200 ps of propagation is needed and still by that time only about $98.5 \%$ of the wavepacket has been absorbed by the CAP. Once the propagations are completed, two procedures can be used to obtain the various state-to-state transition probabilities: it is either the flux ${ }^{20,29,30}$ approach or the Tannor and Weeks ${ }^{31}$ method. We tested both methods but we will mainly present results from the Tannor and Weeks method as both results were similar. Unless specified 
otherwise, in the following equations and text $E=E_{\text {tot }}$ that is the total energy in the collisional process. The kinetic or collision energy will be labelled $E_{k i n}$. Using the Tannor and Weeks method, the state-to-state reaction probabilities are expressed as $^{31,32}$

$$
\begin{aligned}
P_{f \leftarrow i}^{J}(E) & =\left|S_{f i}^{J}(E)\right|^{2} \\
& =\frac{1}{4 \pi^{2}\left|\Delta_{i}(E)\right|^{2}\left|\Delta_{j}(E)\right|^{2}}\left|\int_{0}^{T} e^{i E t} C_{f i}(t) d t\right|^{2}
\end{aligned}
$$

where $S_{f i}$ is the $S$-matrix element, $C_{f i}$ is the autocorrelation function obtained in term of the propagated function $\Psi_{i}$ and the final state function $\Psi_{f}$,

$$
C_{f i}(t)=\left\langle\Psi_{f}\left|e^{-i H t}\right| \Psi_{i}\right\rangle
$$

where $\Delta_{i}$ and $\Delta_{f}$ are the energy distribution ${ }^{33,34}$ of the initial and final wavepackets, respectively. The functions $\Psi_{i}$ and $\Psi_{f}$ are expressed as in Eq. (17) where we can rewrite the labelled transition $i \leftarrow j$ as

$$
j_{1}^{\prime} \zeta_{1}^{\prime} m_{1}^{\prime}, j_{2}^{\prime} l_{2}^{\prime} \leftarrow j_{1} \zeta_{1} m_{1}, j_{2} l_{2}
$$

Here $j_{1}\left(j_{1}{ }^{\prime}\right)$ and $j_{2}\left(j_{2}{ }^{\prime}\right)$ are respectively the orbital angular momentum quantum numbers of the $\mathrm{H}_{2} \mathrm{O}$ and $\mathrm{H}_{2}$ molecule, $\zeta_{1}\left(\zeta_{1}^{\prime}\right)$ and $m_{1}\left(m_{1}^{\prime}\right)$ are respectively an index along the BF-axis which identifies specific rotational states and the Space-Fixed projection of $\mathrm{H}_{2} \mathrm{O}$ running from $-j_{1}$ to $j_{1} . l_{2}\left(l_{2}^{\prime}\right)$ is the Space-Fixed projection of $\mathrm{H}_{2}$ running from $-j_{2}$ to $j_{2}$. In the literature, $\zeta_{1}\left(\zeta_{1}^{\prime}\right)$ is often replaced by $K_{a} K_{c}$ where $K_{a}$ and $K_{c}$ are respectively the projection of the molecular orbital angular momentum along the Body-Fixed (Molecular) axis in the prolate and oblate limits.

As mentioned in a previous publication ${ }^{4}$, while the flux and Tannor and Weeks methods lead to similar probabilities, the flux method usually produces a small, but non-negligible flux in an energetically forbidden region (at energies lower than the total energy threshold). This numerical issue can easily be corrected by zeroing the probabilities in the energetically forbidden region when summing the cross-sections (Eqn. (23)). Conversely, the Tannor and Weeks method requires a broad energy distribution of the final wavefunctions (wavefunctions on which the propagated wavefunction is projected) to make the correlation function disappear more quickly. Following the calculation of the state-to-state transition probabilities, a weighted sum (by the $2 J_{B F}+1$ factor) of these probabilities then produces the inelastic scattering crosssection from the relation

$$
\begin{aligned}
\sigma_{j_{1} \zeta_{1} j_{2}}^{j_{1}^{\prime} \zeta_{1}^{\prime} j_{2}^{\prime}}(E)= & \frac{\pi \hbar^{2}}{2 \mu_{R}\left(2 j_{1}+1\right)\left(2 j_{2}+1\right) E_{k i n}} \times \\
& \sum_{m_{1}=-j_{1}}^{j_{1}} \sum_{l_{2}=-j_{2}}^{j_{2}} \sum_{m_{1}^{\prime}=-j_{1}^{\prime}}^{j_{1}^{\prime}} \sum_{l_{2}^{\prime}=-j_{2}^{\prime}}^{j_{2}^{\prime}} \sum_{J_{B F}=0}^{J_{\max }} \times \\
& \left(2 J_{B F}+1\right) P_{j_{1}^{\prime} \zeta_{1}^{\prime} m_{1}^{\prime}, j_{2}^{\prime} l_{2}^{\prime} \leftarrow j_{1} \zeta_{1} m_{1}, j_{2} l_{2}}^{J_{B F}}(E),
\end{aligned}
$$

where $E_{k i n}=E-E_{\text {int }}=E-\epsilon_{H_{2}}-\epsilon_{H_{2} O}$ with $E_{\text {int }}, \epsilon_{H_{2}}$ and $\epsilon_{\mathrm{H}_{2} \mathrm{O}}$ the internal initial energy of the whole system and the initial rotational energies of the $\mathrm{H}_{2}$ and $\mathrm{H}_{2} \mathrm{O}$ fragments respectively.

\section{RESULTS AND DISCUSSIONS}

Before presenting and discussing the results, it is worth pointing out that the purpose of this work was not simply to demonstrate precise agreement between the MCTDH results and those obtained with the Time-Independent Close-Coupling method (if any could be found), but rather to highlight the computational efficiency with which MCTDH makes it possible to obtain accurately the cross section with reasonably limited resources. However, as the following results will show, we were able to obtain overall a good agreement with Close Coupling results. For all of the calculations reported in this work we used a Primitive and Single Particle Functions basis as reported in Table II. Between 1,000 to 6,000 Single Particle Functions were used to describe the dynamics of the system compared with more than 1,000,000 Primitive Basis functions that would have been needed overall for a standard wave packet calculation.

\section{A. Convergence of the calculations}

The convergence of the MCTDH calculations depends on various parameters: the primitive basis, the SPF basis, the range of the propagation, the intensity of the CAP and the duration of the propagation. For this work we used a primitive basis with angular parameters similar to the one used by Scribano et $a l^{15}$ : the number of DVR points for the radial degree of freedom was selected to be sufficiently dense for the range selected.

\section{Convergence: radial coordinate range}

To determine the range of integration, we performed 2 scattering calculations: one going from 2 to 52 bohrs (with 768 radial DVR points) and the other from 2 to 72 bohrs (with 960 radial DVR points), that is with about the same density of points along the radial coordinate for the 2 calculations, but also the same number of SPF (1,000 SPF for these tests). Those 2 calculations were compared with the available close-coupling calculations (considered exact) as depicted in Figure 1. As expected, extending the grid and displacing the position of the CAP (which however keeps the same length) improves the quality of the results. Also, the contraction of the grid induces a very small blue shift in the position of the resonance structures of the cross-section. We computed the transition probabilities for each individual $J$ from 0 to 35 (the maximum $J$ value considered for the time independent close coupling calculations) in parallel using 
FIG. 1. Comparison of MCTDH calculations for $R_{\max }=52$ and $R_{\max }=72$ with time independent close coupling results for the $0_{00} \rightarrow 1_{11}$ rotational transition of $\mathrm{H}_{2} \mathrm{O}$.

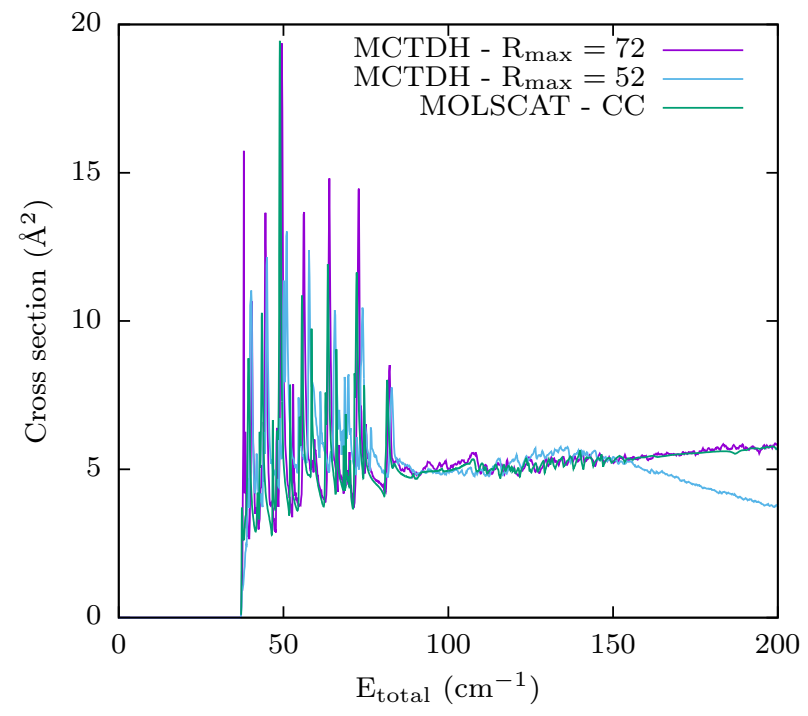

32 processors for each $J$-calculation. These calculations with 1,000 SPF lasted from less than a day for the higher $J$-values to about 7 days for the lower $J$-values which require a longer propagation.

\section{Convergence: the energy distribution}

An important aspect of the calculation is the choice of the initial wavefunction. As stated before, the width and initial momentum of the Gaussian are connected to the energy range that will be covered by the scattering calculation. We perform 2 separate scattering calculations to see the influence of the final cross-sections on the choice of the energy range covered by the scattering. For the first calculation we selected a Gaussian centered at 52 bohr with a width of 1.2 a.u. and an initial momentum of -1.6 a.u. (where the negative sign emphasizes the fact that the initial wave packet goes towards the interaction region) and for the second calculation, the Guassian is centered at 52 bohr but with a width of 1.0 a.u. and an initial momentum of -2.1 a.u. The energy distribution of the 2 calculations at $J=0$ are displayed in Figure 2 and a comparison between the cross-section for the $0_{00} \rightarrow 1_{11}$ transition are compared with the time independent close coupling calculation on Figure 3. The comparison shows that the calculation with the shorter energy distribution range describes better the low energy than one with the larger range. Therefore, for calculations spanning a large collisional energy range, it would be necessary to perform different sets of calculations with two or more energy distribution ranges in order to cover accurately a wide range of collision energies.
FIG. 2. Energy distribution used for the MCTDH calculations at $J=0$. edist1 is generated with a Gaussian of width 1.2 a.u. and an initial momentum of -1.6 a.u. and edist 2 with a Gaussian of width 1.0 a.u. and momentum of -2.1 a.u.

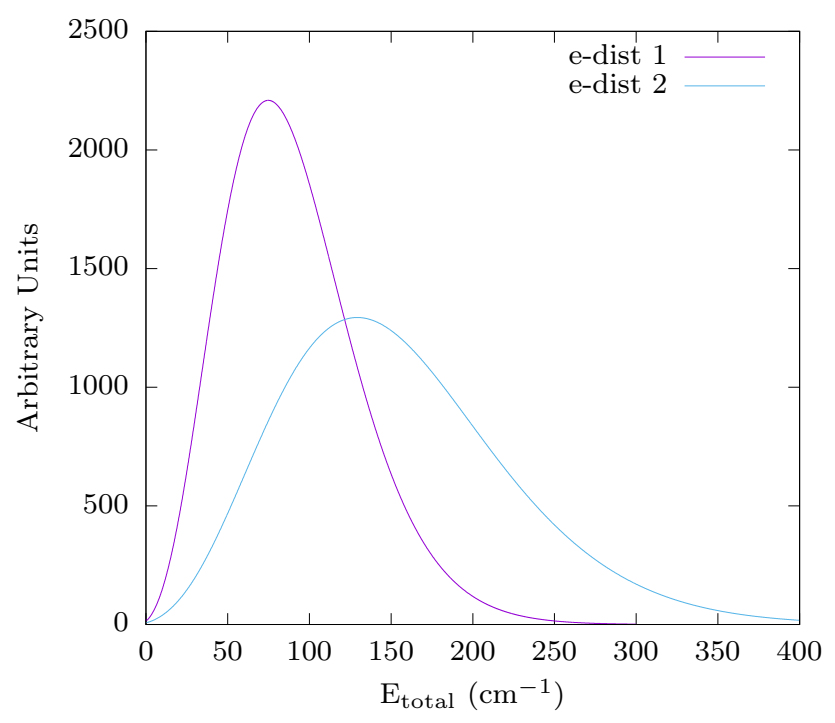

FIG. 3. Comparison of MCTDH calculations for edist1 and edist2 with time independent close coupling results for the $0_{00} \rightarrow 1_{11}$ rotational transition of $\mathrm{H}_{2} \mathrm{O}$.

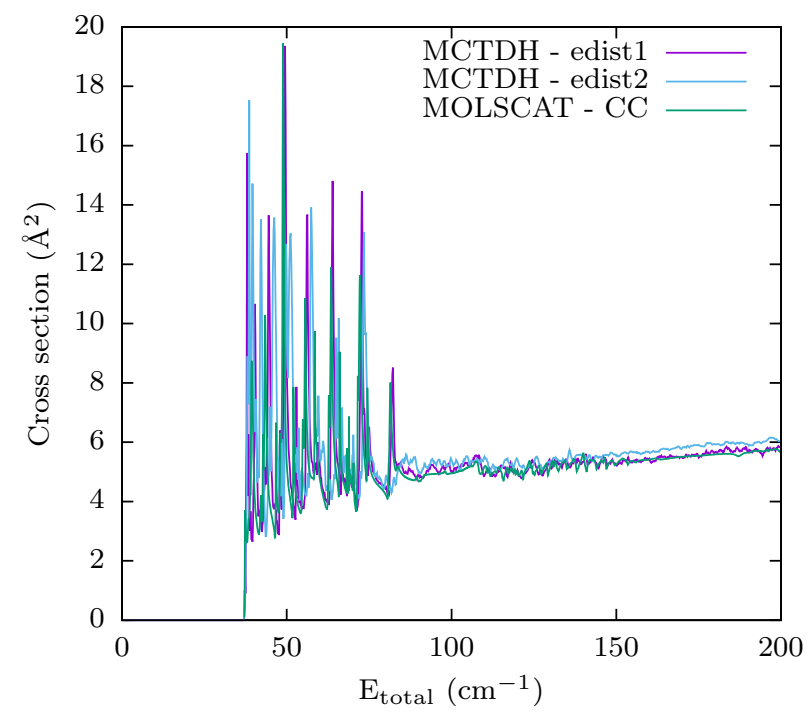

\section{Convergence: the SPF basis}

An advantage of the MCTDH method is that the approach, just like the Close Coupling method, is variational. Therefore, we know that once a suitable choice of a primitive basis is made, we can improve the accuracy of the calculation by further increasing the size of the SPF basis. A characteristic of the MCTDH method is that it is designed to reproduce very well the most intense fea- 
FIG. 4. Comparison between MCTDH calculations with 4 different set of basis and Exact (MOLSCAT) time independent close coupling calculations. Each MCTDH calculations is shifted by $0.2 \AA^{2}$ downward with respect to the previous one and the most accurate calculation is closer to the MOLSCAT result. The parameters of the basis are given in Table III.

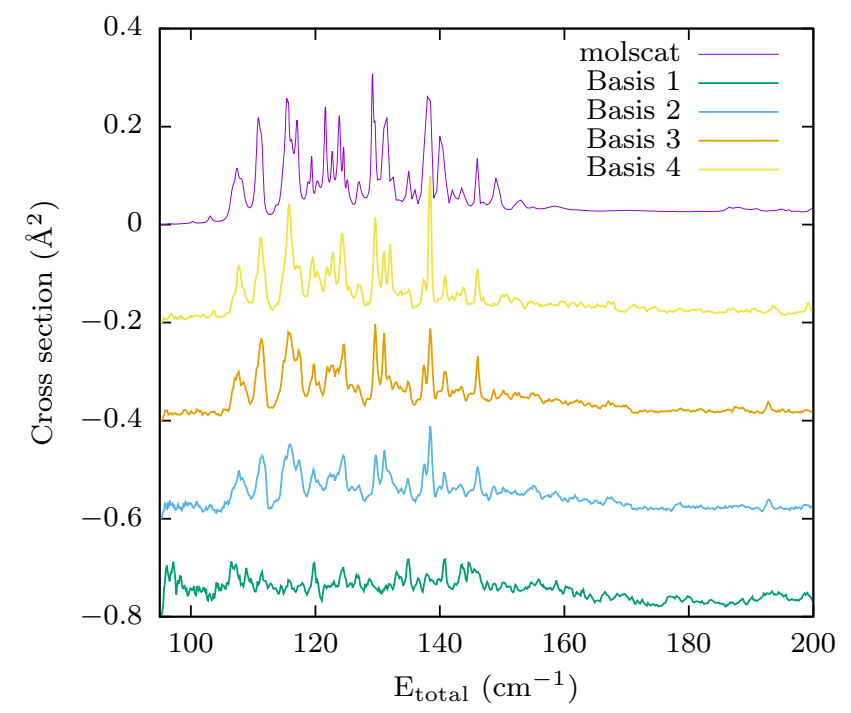

TABLE III. Parameters of the SPF basis used in Figure 4.

\begin{tabular}{ccccc}
\hline & Basis 1 & Basis 2 & Basis 3 & Basis 4 \\
\hline$n_{R}$ & 10 & 12 & 15 & 20 \\
$n_{\beta, k_{\gamma}, k_{\alpha}}$ & 10 & 15 & 20 & 25 \\
$n_{\tilde{\theta}, k_{\phi}}$ & 10 & 10 & 12 & 12 \\
\hline
\end{tabular}

tures of a process with a limited number of functions: however, the less intense features can be recovered easily by increasing the size of the Single Particle Functions. In the limit $N_{S P F} \rightarrow N_{P B F}$, we can recover the exact numerically exact calculations, where $N_{S P F}$ and $N_{P B F}$ are the total number of Single Particle and Primitive Basis Functions respectively. We were able to verify this feature by looking specifically at the $0_{00} \rightarrow 2_{11}$ transition with an increasing SPF basis, and comparing the results with the exact Time Independent results. One aspect of that transition is that it is about 50 times less intense than the $0_{00} \rightarrow 1_{11}$ transition which is the most intense one, and also retrieved from the same set of calculations. The comparison is shown in Figure 4. The parameters of the 4 basis are presented in Table III for the 3 combined modes and are such that the larger combination of SPF basis is the one mentioned in the Table, but for calculation a higher $J$, the combination of numbers is likely to be smaller. For the final results reported in this work, we had to decide on convergence criteria. As we had to do several propagations (for each $J$ ), and since the SPF basis size decreases as $J$ increases, we chosed an SPF basis such that for each (combined) mode, the weight of the last SPF (arranged in decreasing order of weight) should
FIG. 5. Comparison of MCTDH calculations with time independent close coupling results for the $0_{00} \rightarrow 1_{11}$ rotational transition of $\mathrm{H}_{2} \mathrm{O}$.

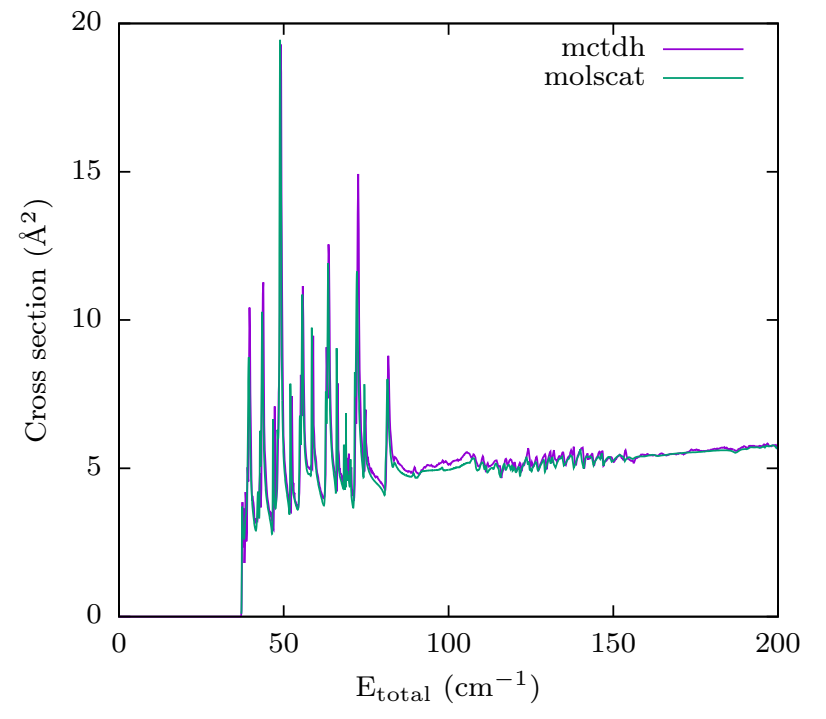

be less than $10^{-5}$ for all the calculations. This uniform criteria allowed to varying basis which will however maintain a consistent accuracy of the calculation.

\section{B. Rotational excitation cross sections}

The calculated inelastic scattering cross-sections for the transitions from the ground state to the first excited states, i.e. $0_{00} \rightarrow 1_{11}, 0_{00} \rightarrow 2_{02}$ and $0_{00} \rightarrow 2_{11}$ are displayed on Figures 5, 6 and 7 respectively. The figures show a very good agreement between the MCTDH calculations and the CC calculations at these low energies. In particular, the position of the resonances are well reproduced in each calculations and an even better agreement will certainly be met with a larger SPF basis. The position and the intensity/width of the peaks are connected in the calculations to the range and the duration of the propagation respectively. A too-narrow range of the propagation shifts the peaks to higher energies because of confinement effects and a shorter duration of the propagation would make the resonances too broad or even nonexistent. In fact, we actually see in the course of the propagation that some resonances start to appear only after a specific amount of propagation time has elapsed. Similarly, just like with a rovibrational calculation, increasing the size of the (SPF) basis improves the agreement on the peaks position with the time independent calculations which are considered here to be numerically exact.

Similarly to the calculations involving the para-states, we perfomed calculations for the ortho-states. Here however, as we mentiond before, we used the same basis sets (made of both para and ortho rotational states 
FIG. 6. Comparison of MCTDH calculations with time independent close coupling results for the $0_{00} \rightarrow 2_{02}$ rotational transition of $\mathrm{H}_{2} \mathrm{O}$.

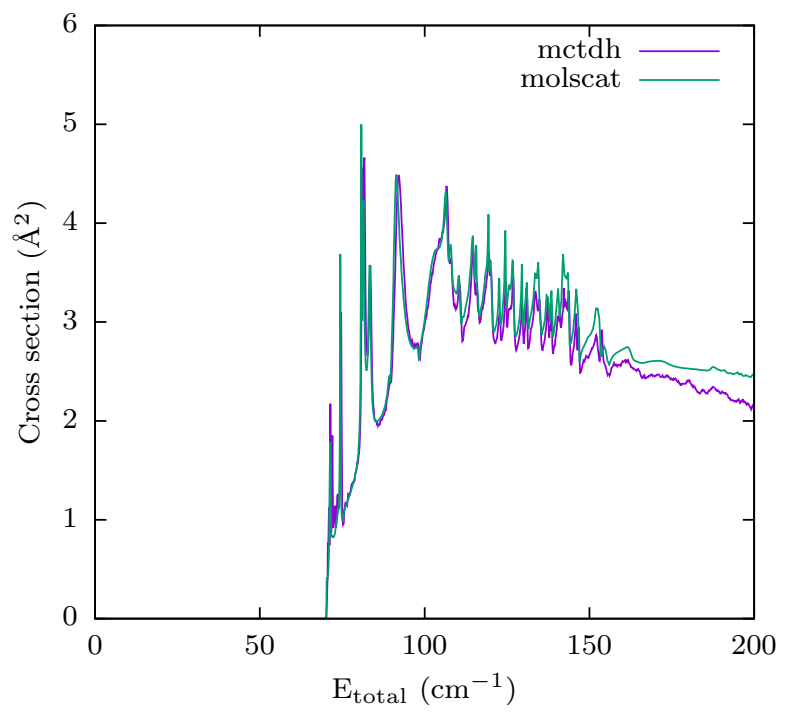

FIG. 7. Comparison of MCTDH calculations with time independent close coupling results for the $0_{00} \rightarrow 2_{11}$ rotational transition of $\mathrm{H}_{2} \mathrm{O}$.

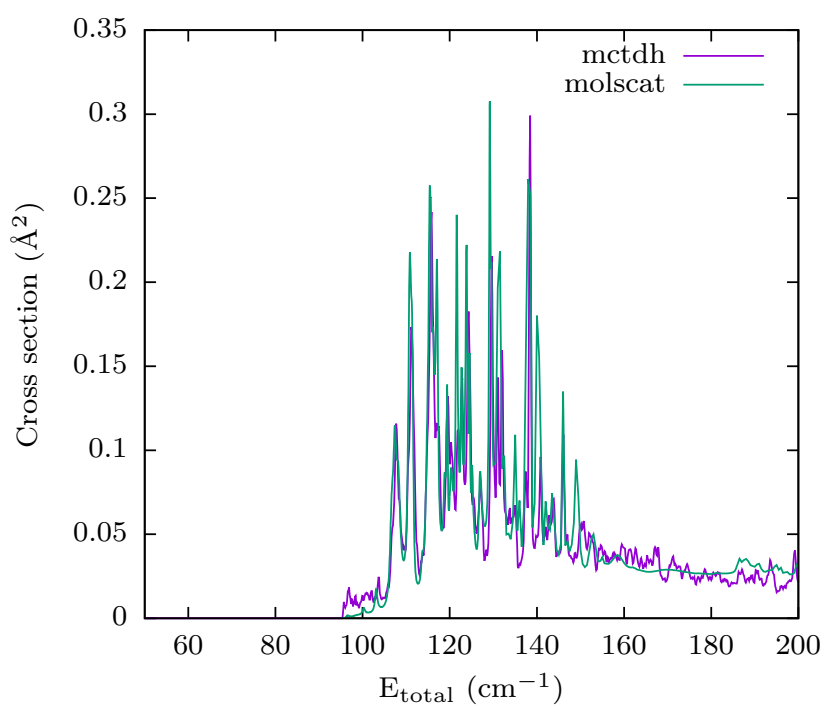

of $\mathrm{H}_{2} \mathrm{O}$ ) to run the propagation and obtain the crosssections. The excitattion cross-sections for 2 ortho transitions $1_{01} \rightarrow 1_{10}$ and $1_{01} \rightarrow 2_{12}$ are displayed respectively in Figures 8 and 9 . Here again, we observe a very good agreement between the MCTDH and the time independent results, both with respect to the position and the intensity of the peaks. We notice however some small issues at the start of the cross-sections where some peaks are displayed with a smaller intensity than the time independent results, an issue that is likely linked to the
FIG. 8. Comparison of MCTDH calculations with time independent close coupling results for the $1_{01} \rightarrow 1_{10}$ rotational transition of $\mathrm{H}_{2} \mathrm{O}$.

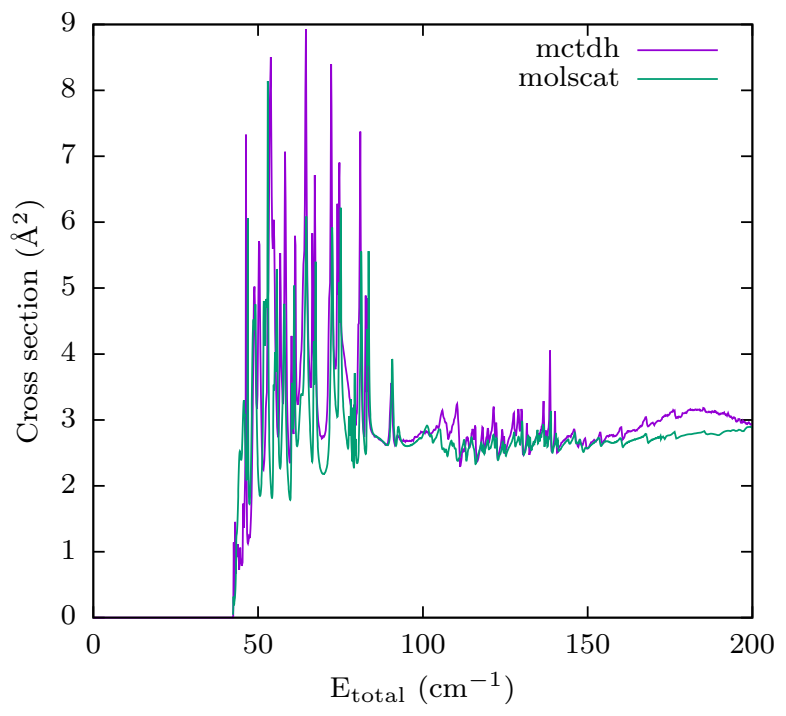

FIG. 9. Comparison of MCTDH calculations with time independent close coupling results for the $1_{01} \rightarrow 2_{12}$ rotational transition of $\mathrm{H}_{2} \mathrm{O}$.

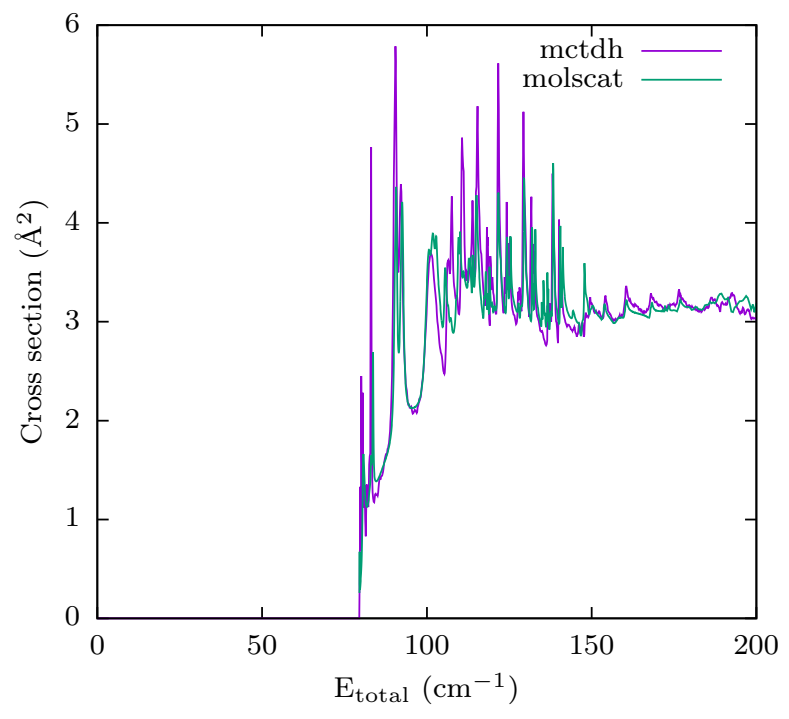

duration of the propagation. Also towards the end of the spectrum, it appears that the MCTDH calculations have a higher amplitude than the time independent results, an issue that would be corrected if a wider energy distribution appropriate to describe higher energy values were to be selected. 
FIG. 10. Comparison of MCTDH calculations with time independent close coupling results for the $1_{11} \rightarrow 0_{00}$ rotational transition of $\mathrm{H}_{2} \mathrm{O}$.

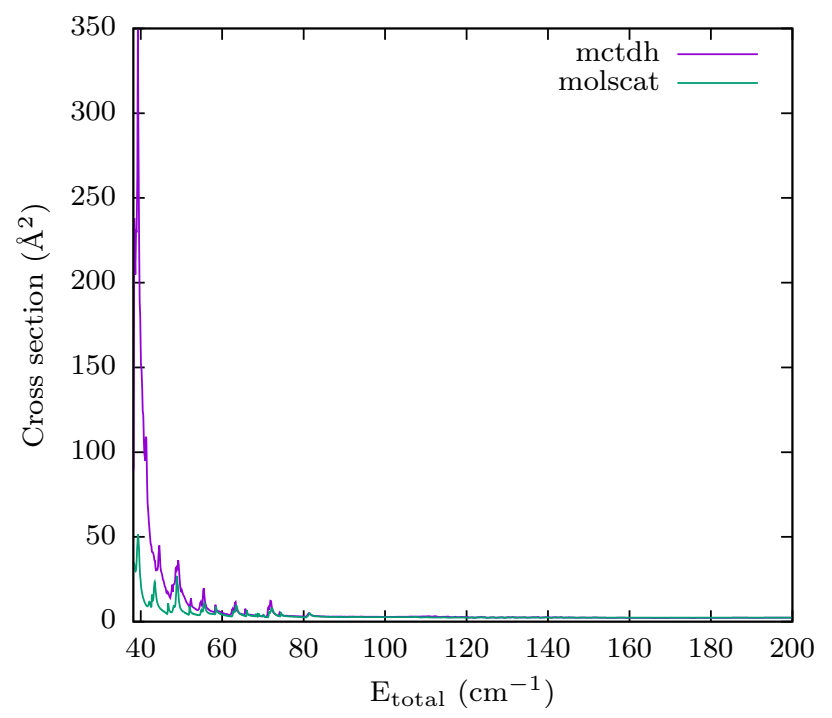

\section{Rotational de-excitation cross sections}

We performed additional calculations to determine the state-to-state cross-sections from the first excited parastate. This allowed to obtain a de-excitation cross-section $1_{11} \rightarrow 0_{00}$ in addition to an excitation cross-section $1_{11} \rightarrow 2_{02}$. These cross-sections are displayed in Figures 10 and 12 . Figure 11 is similar to Figure 10 with however a logarithmic scale used for the $y$-axis (crosssections intensity). We observe as in the previous cases that the excitation cross-section reproduces quite well the position of the peaks/resonances of the spectrum; this is also the case for the de-excitation process until we get to very low kinetic energy values. However, while the intensities are usually quite well reproduced for the excitation processes, the logarithmic scale cross-sections (Figure 11) highlights how difficult it is to accomplish it for the de-excitation process. This difference in accuracy between the 2 types of calculations (excitation and deexcitation) using the same SPF basis can be understood from the cross-sections equation (Eq. (23)) but also from the calculation of state-to-state transition probabilities in the flux formalism. Within this formalism, the transition probabilities are obtained as a ratio of the flux going towards a specific channel to the total flux. When the calculation is exact, the total flux is well approximated by the energy distribution of the propagation. While for the excitation processes the starting point of the state-to-state cross-sections is often in an energy region where the absolute value of the energy distribution is quite significant, for de-excitation processes, this often corresponds to an energy region where the energy distribution is very small, 2 order of magnitude or more lower than the peak of the energy distribution. As the
FIG. 11. Comparison of MCTDH calculations with time independent close coupling results for the $1_{11} \rightarrow 0_{00}$ rotational transition of $\mathrm{H}_{2} \mathrm{O}$. Note the logscale applied to the $y-$ axis (cross-section intensity).

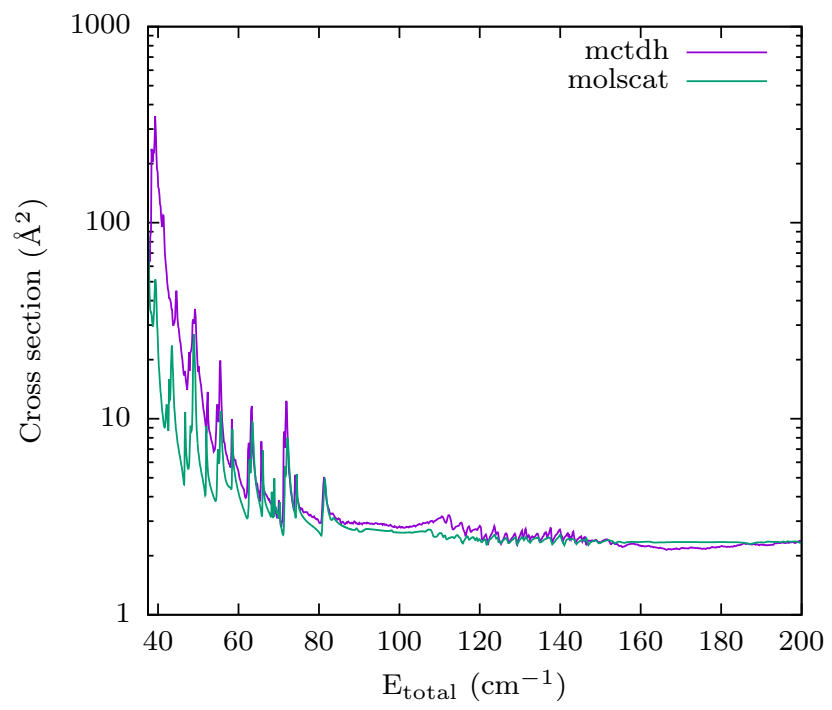

FIG. 12. Comparison of MCTDH calculations with time independent close coupling results for the $1_{11} \rightarrow 2_{02}$ rotational transition of $\mathrm{H}_{2} \mathrm{O}$.

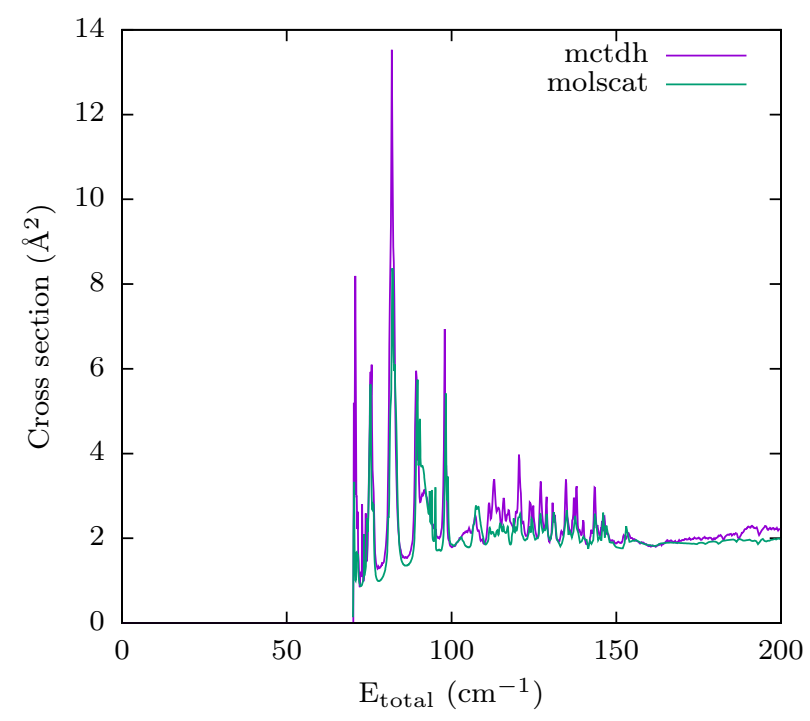

flux to that channel at low kinetic energy is also very small, it is quite difficult to describe with a small contracted basis designed for the whole energy range, the small features that mainly influence the low kinetic energy domain. Additionally, the $1 / E_{c}$ term amplifies the disagreement between the MCTDH and time independent calculations at low collision energies where its role is alleviated as energy increases as we can see from the picture. However, we realized that by increasing the SPF 
FIG. 13. Comparison of MCTDH calculations-with the smaller (old) and larger (new) basis- with time independent close coupling results for the $1_{11} \rightarrow 0_{00}$ rotational transition of $\mathrm{H}_{2} \mathrm{O}$. Note the logscale applied to the $y$-axis.

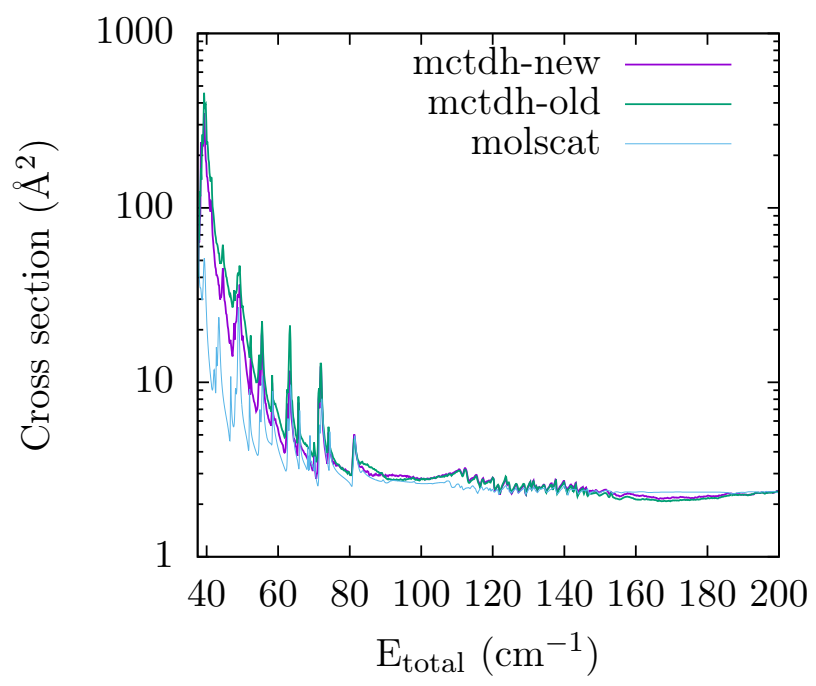

basis size we were able to significantly improve the comparison with the time independent results. For example, while for most of the calculations displayed in this work we used a SPF basis with parameters described in Table II, for the $1_{11} \rightarrow 0_{00}$ we used up to 35 SPFs for the $\mathrm{H}_{2} \mathrm{O}$ angular basis and up to $25 \mathrm{SPFs}$ for the FFT on the $R$ coordinate on some $J$ propagation that we identified as the most problematic. Despite this significant increase, we noted some (modest) change on the low energy region of the de-excitation cross-section for the $1_{11} \rightarrow 0_{00}$ transition while only a modest to nonexistant one on the excitation cross-section for the $1_{11} \rightarrow 2_{02}$ transition as we can see on Figures 13 and 14 respectively. So essentially we only observe a problem in the de-excitation cross-section of the $1_{11} \rightarrow 0_{00}$ transition within $10-20$ $\mathrm{cm}^{-1}$ above the treshold energy, i.e. here, below $\approx 55$ $\mathrm{cm}^{-1}$ of total energy $E$.

\section{CONCLUSION}

We performed the first benchmark 5D calculations for the inelastic scattering of an asymmetric top molecule $\left(\mathrm{H}_{2} \mathrm{O}\right)$ with a linear molecule $\left(\mathrm{H}_{2}\right)$, all in the rigid rotor approximation, using the MultiConfiguration Time Dependent Hartree procedure and without any additional approximation on a recently constructed PES. We find as expected a very good agreement between the time independent close coupling results and our calculations which are done with a relatively modest amount of resources.

Time Independent Close Coupling calculations are frequently regarded as the benchmark for these type of atom/molecule or molecule/molecule collisional pro-
FIG. 14. Comparison of MCTDH calculations-with the smaller (old) and larger (new) basis-with time independent close coupling results for the $1_{11} \rightarrow 2_{02}$ rotational transition of $\mathrm{H}_{2} \mathrm{O}$.

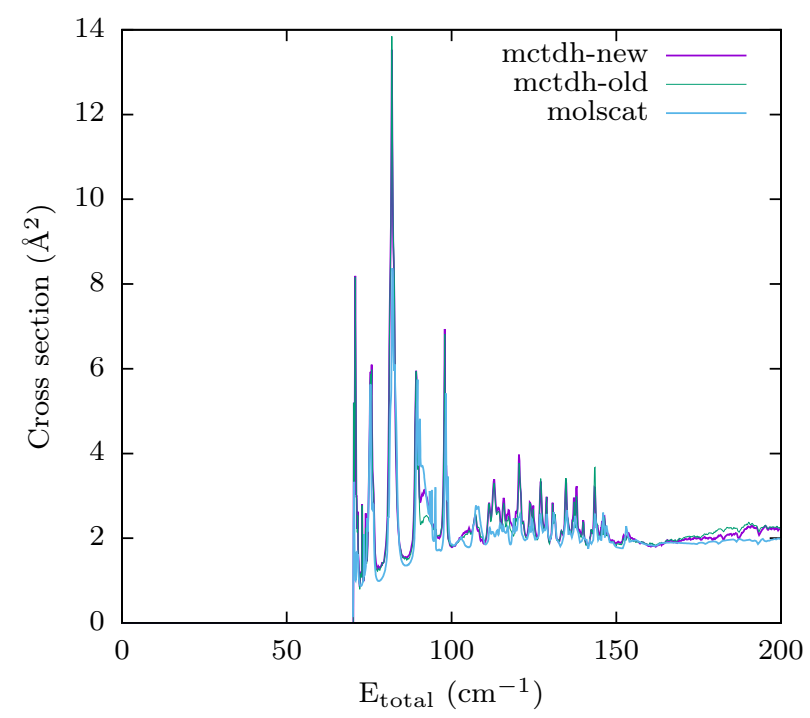

cesses. However, they quickly become untractable when either the density of rotational states of the partners or the dimensionality of the collisional system increases. It then becomes essential to look for alternate means of computing collisional cross-sections. We anticipate that the MCTDH procedure (and its variants ML-MCTDH, ...) could be one of those alternate methods to describe fully quantum mechanically the collisional dynamics in these challenging cases. As the MCTDH inelastic scattering calculations usually generate cross-sections for all transitions starting from an initial state, they will also be very relevant for comparison with experimental results where quite often only selected transitions could be measured.

With respect to astrophysical applications where one is often interested in the state-to-state rates, instead of the specific state-to-state cross-sections, one can infer the state-to-state rate of a de-excitation process (where the cross-sections calculations appear to be challenging at low kinetic energy using the MCTDH method) from the reverse excitation process, which is known as detailed balance.

The current MCTDH implementation of the WignerDVR does not yet enable even/odd symmetry specified calculations. Hence it was not possible to selectively perform wavepacket propagation calculations for the para and ortho cases which have a specific symmetry with respect to the $\mathrm{H}_{2} \mathrm{O}$ fragment axis. We thus did not take advantage of the primitive basis size reductions that would occur from the symmetry consideration. Nevertheless, the calculations for this system are still tractable with respect to both the time and memory requirements.

The usage of the MCTDH method for this type of ap- 
plications is still at a development stage. However, we are working on defining a scheme for which these type of computations are likely to become standard and straightforward for people interested in such applications. Imposing the symmetry for the Wigner-DVR in the MCTDH package will be part of this process and will certainly improve the results.

\section{ACKNOWLEDGMENTS}

This research was supported by the U.S. department of Energy Office of Science, Office of Basic Energy Sciences [Award No. DE-SC0010616] to R.D. S.A.N thanks Hans-Dieter Meyer for various discussions related to the MCTDH implementation of this calculation. Y.S thanks Alexandre Faure for providing us the close-coupling data.

${ }^{1}$ A. Semenov and D. Babikov, J. Phys. Chem. Lett. 6, 1854 (2015).

${ }^{2}$ J. Loreau, A. Faure, and F. Lique, J. Chem. Phys. 148, 244308 (2018).

${ }^{3}$ J. Loreau, F. Lique, and A. Faure, Astrophys. J. Lett. 853, L5 (2018).

${ }^{4}$ S. Ndengué, R. Dawes, F. Gatti, and H. D. Meyer, Chem. Phys. Lett. 668, 42 (2017).

${ }^{5}$ A. van der Avoird and D. Nesbitt, J. Chem. Phys. 134, 044314 (2011).

${ }^{6}$ X.-G. Wang and T. Carrington Jr., J. Chem. Phys. 134, 044313 (2011).

${ }^{7}$ Y. Scribano, A. Faure, and D. Lauvergnat, J. Chem. Phys. 136, 094109 (2012).

${ }^{8}$ Y. Scribano and A. Faure, J. Chem. Phys. 146, 226102 (2017).

${ }^{9}$ S. A. Ndengue, R. Dawes, F. Gatti, and H. Guo, J. Phys. Chem. A 122, 6381 (2018).

${ }^{10}$ T. R. Philips, S. Maluendes, and S. Green, J. Chem. Phys. 102, 6024 (1995).

${ }^{11}$ M. L. Dubernet and A. Grosjean, Astron. \& Astrophys. 390, 793 (2002).

${ }^{12}$ A. Grosjean, M. L. Dubernet, and C. Ceccarelli, J. Chem. Phys. 408, 1197 (2003).
${ }^{13}$ M. L. Dubernet, F. Daniel, A. Grosjean, A. Faure, P. Valiron, M. Wernli, L. Wiesenfeld, C. Rist, J. Noga, and J. Tennyson, Astron. \& Astrophys. 460, 323 (2006).

${ }^{14}$ M. L. Dubernet, F. Daniel, A. Grosjean, and C. Y. Lin, Astron. \& Astrophys. 497, 911 (2009).

${ }^{15}$ Y. Scribano, A. Faure, and L. Wiesenfeld, J. Chem. Phys. 133, 231105 (2010).

${ }^{16}$ A. Faure, N. Crimier, C. Ceccarelli, P. Valiron, L. Wiesenfeld, and M. L. Dubernet, Astron. \& Astrophys. 472, 1029 (2007).

${ }^{17}$ H.-D. Meyer, U. Manthe, and L. S. Cederbaum, Chem. Phys. Lett. 165, 73 (1990).

${ }^{18}$ U. Manthe, H.-D. Meyer, and L. S. Cederbaum, J. Chem. Phys. 97, 3199 (1992).

${ }^{19}$ H. D. Meyer, F. Gatti, and G. A. Worth, eds., Multidimensional Quantum Dynamics: MCTDH Theory and Applications (WileyVCH: Weinheim, 2009).

${ }^{20}$ M. H. Beck, A. Jäckle, G. A. Worth, and H. D. Meyer, Phys. Rep. 324, 1 (2000).

${ }^{21}$ S. Ndengué, Y. Scribano, D. M. Benoit, F. Gatti, and R. Dawes, Chem. Phys. Lett. 715, 347 (2018).

${ }^{22}$ G. Brocks, A. V. D. Avoird, B. T. Sutcliffe, and J. Tennyson, Mol. Phys. 50, 1025 (1983).

${ }^{23}$ F. Gatti and C. Iung, Phys. Rep. 484, 1 (2009).

${ }^{24}$ C. Leforestier, J. Chem. Phys. 101, 7357 (1994).

${ }^{25}$ P. Valiron, M. Wernli, A. Faure, L. Wiesenfeld, C. Rist, S. Kedzuch, and J. Noga, J. Chem. Phys. 129, 134306 (2008).

${ }^{26}$ S. A. Ndengue, R. Dawes, and F. Gatti, J. Phys. Chem. A 119, $7712(2015)$.

${ }^{27}$ A. Meurer, C. P. Smith, M. Paprocki, O. Čertík, S. B. Kirpichev, M. Rocklin, A. Kumar, S. Ivanov, J. K. Moore, S. Singh, T. Rathnayake, S. Vig, B. E. Granger, R. P. Muller, F. Bonazzi, H. Gupta, S. Vats, F. Johansson, F. Pedregosa, M. J. Curry, A. R. Terrel, v. Roučka, A. Saboo, I. Fernando, S. Kulal, R. Cimrman, and A. Scopatz, PeerJ Computer Science 3, e103 (2017).

${ }^{28}$ S. Sukiasyan and H. D. Meyer, J. Chem. Phys. 116, 10641 (2002).

${ }^{29}$ T. Seideman and W. H. Miller, J. Chem. Phys. 96, 4412 (1992).

${ }^{30}$ A. Jäckle and H.-D. Meyer, J. Chem. Phys. 105, 6778 (1996).

${ }^{31}$ D. J. Tannor and D. E. Weeks, J. Chem. Phys. 98, 3884 (1993).

${ }^{32}$ A. N. Panda, F. Otto, F. Gatti, and H.-D. Meyer, J. Chem. Phys. 127, 114310 (2007).

${ }^{33}$ F. Gatti, F. Otto, S. Sukiasyan, and H. D. Meyer, J. Chem. Phys. 123, 174311 (2005).

${ }^{34}$ A. Jäckle and H. D. Meyer, J. Chem. Phys. 109, 2614 (1998). 